Louisiana State University

LSU Digital Commons

Faculty Publications

Department of Mathematics

6-1-2001

\title{
The itô table of the square of white noise
}

\author{
Luigi Accardi \\ Università degli Studi di Roma Tor Vergata \\ Takeyuki Hida \\ International Institute for Advanced Studies \\ Hui Hsiung Kuo \\ Louisiana State University
}

Follow this and additional works at: https://digitalcommons.Isu.edu/mathematics_pubs

\section{Recommended Citation}

Accardi, L., Hida, T., \& Kuo, H. (2001). The itô table of the square of white noise. Infinite Dimensional Analysis, Quantum Probability and Related Topics, 4 (2), 267-275. https://doi.org/10.1142/ S0219025701000437

This Article is brought to you for free and open access by the Department of Mathematics at LSU Digital Commons. It has been accepted for inclusion in Faculty Publications by an authorized administrator of LSU Digital Commons. For more information, please contact ir@lsu.edu. 
See discussions, stats, and author profiles for this publication at: http://www.researchgate.net/publication/263914484

\section{The Itô Table of the Square of White Noise}

ARTICLE in INFINITE DIMENSIONAL ANALYSIS QUANTUM PROBABILITY AND RELATED TOPICS · NOVEMBER 2011

Impact Factor: $0.73 \cdot$ DOI: 10.1142/S0219025701000437

CITATIONS

10

3 AUTHORS, INCLUDING:

Luigi Accardi

University of Rome Tor Vergata

466 PUBLICATIONS 2,555 CITATIONS

SEE PROFILE
READS

5 


\title{
THE ITÔ TABLE OF THE SQUARE OF WHITE NOISE
}

\author{
LUIGI ACCARDI \\ Centro Vito Volterra, Facoltà di Economia, Università di Roma Tor Vergata, \\ Via di Tor Vergata, 00133 Roma, Italy \\ TAKEYUKI HIDA \\ International Institute for Advanced Studies, 9-3 Kizugawadai, \\ Kizu, Soraku-gun, Kyoto 619-0225, Japan \\ HUI-HSIUNG KUO \\ Department of Mathematics, Louisiana State University, \\ Baton Rouge, LA 70803-4918, USA
}

In a recent paper [3] Accardi, Lu and Volovich have introduced the $*$-algebra of the renormalized square of white noise (SWN). This algebra is generated by the operators $B_{f}, B_{f}^{\dagger}, N_{f}$, whose formal expressions, in terms of the square of the standard quantum white noise $b_{t}^{+}, b_{t}$ are:

$$
B_{f}=\int b_{t}^{2} \bar{f}(t) d t, \quad B_{f}^{\dagger}=\int b_{t}^{\dagger 2} f(t) d t, \quad N_{f}=\int b_{t}^{\dagger} b_{t} f(t) d t
$$

with the following commutation relations:

$$
\begin{aligned}
{\left[B_{f}, B_{g}^{\dagger}\right] } & =2 c(f, g)+4 N_{\bar{f} g}, \\
{\left[N_{f}, B_{g}^{\dagger}\right] } & =2 B_{f g}^{\dagger}, \\
{\left[N_{f}, B_{g}\right] } & =-2 B_{\bar{f} g}, \\
{\left[B_{f}, B_{g}\right] } & =\left[B_{f}^{\dagger}, B_{g}^{\dagger}\right]=\left[N_{f}, N_{g}\right]=0, \\
B_{f} \Phi & =N_{f} \Phi=0,
\end{aligned}
$$

where $c$ is a strictly positive number, $(f, g)=\int \bar{f}(t) g(t) d t$ (coming from renormalization), and $\Phi$ is the vacuum vector.

The SWN algebra was extended to the free case in [8]. The exponential vectors for the SWN were introduced in [5] where the connection with Boukas-Feinsilver's finite difference algebra was first noticed. As shown in [1], the deep roots of these connections are to be found in the theory of representation of current algebras and of quantum independent increment processes. This general approach has allowed to construct infinitely many representations of the SWN, inequivalent to the Fock one and to obtain similar representations for the higher powers of the white noise. In particular the results of [1] allow to reduce 
completely the stochastic integration theory for the SWN, obtained with direct estimates in [9], to the usual Hudson-Parthasarathy stochastic integration. However this reduction does not seem to solve in a simple way (at least at the present moment) the problem of deciding if and in what sense the basic integrators of the SWN have a closed Ito table. In the present note we show, by direct methods, that the answer to the above question is affirmative for the second order Ito table. More precisely, our main result is the following:

Theorem (1). The mutual quadratic variation of any pair of the basic integrators of the $S W N$ (cf. (9c) for their definition) exists in the topology of weak convergence of the matrix elements in the $S W N$-exponential vectors with test functions satisfying the conditions (9a), (9b) below.

Due to the factorization property of the space of the SWN (cf. [5] and [1]), the second order Ito table is sufficient to establish the unitarity condition (which will be discussed in another paper). The topology used in Theorem (1) is too weak to guarantee the closure of the Ito table at any order. We conjecture, that this is the case, but the proof of this statement requires a further development of the theory.

We will use some known properties of the action of $B_{f}$ and $N_{f}$ on the exponential vectors of the SWN which, for completeness, we prove in the following three lemmata. Recall from [5] that an exponential vector for the SWN is a vector of the form

$$
\psi(g)=\sum_{n=0}^{\infty} \frac{1}{n !}\left(B_{g}^{\dagger}\right)^{n} \Phi
$$

Lemma 1. For any $n \geq 1, N_{f}\left(B_{g}^{\dagger}\right)^{n} \Phi=2 n B_{f g}^{\dagger}\left(B_{g}^{\dagger}\right)^{n-1} \Phi$.

Proof. By Equations (2), (4) and (5) we get

$$
\begin{aligned}
N_{f}\left(B_{g}^{\dagger}\right)^{n} \Phi & =\left\{\left[N_{f},\left(B_{g}^{\dagger}\right)^{n}\right]+\left(B_{g}^{\dagger}\right)^{n} N_{f}\right\} \Phi=\left[N_{f},\left(B_{g}^{\dagger}\right)^{n}\right] \Phi \\
& =\sum_{j=0}^{n-1}\left(B_{g}^{\dagger}\right)^{j}\left[N_{f}, B_{g}^{\dagger}\right]\left(B_{g}^{\dagger}\right)^{n-1-j} \Phi \\
& =\sum_{j=0}^{n-1}\left(B_{g}^{\dagger}\right)^{j}\left(2 B_{f g}^{\dagger}\right)\left(B_{g}^{\dagger}\right)^{n-1-j} \Phi=2 n B_{f g}^{\dagger}\left(B_{g}^{\dagger}\right)^{n-1} \Phi
\end{aligned}
$$

Lemma 2. $N_{f} \psi(g)=2 B_{f g}^{\dagger} \psi(g)$.

Proof. Apply Lemma 1 and use Equation (5) to get

$$
N_{f} \psi(g)=\sum_{n=1}^{\infty} \frac{1}{n !} N_{f}\left(B_{g}^{\dagger}\right)^{n} \Phi=\sum_{n=1}^{\infty} \frac{1}{n !} 2 n B_{f g}^{\dagger}\left(B_{g}^{\dagger}\right)^{n-1} \Phi=2 B_{f g}^{\dagger} \psi(g)
$$

Lemma 3. $B_{f} \psi(g)=\left(2 c(f, g)+2 N_{\bar{f} g}\right) \psi(g)$. 
Proof. First apply Equations (1) and (5) to get

$$
\begin{aligned}
B_{f}\left(B_{g}^{\dagger}\right)^{n} \Phi & =\left\{\left[B_{f},\left(B_{g}^{\dagger}\right)^{n}\right]+\left(B_{g}^{\dagger}\right)^{n} B_{f}\right\} \Phi=\left[B_{f},\left(B_{g}^{\dagger}\right)^{n}\right] \Phi \\
& =\sum_{j=0}^{n-1}\left(B_{g}^{\dagger}\right)^{j}\left[B_{f}, B_{g}^{\dagger}\right]\left(B_{g}^{\dagger}\right)^{n-1-j} \Phi \\
& =\sum_{j=0}^{n-1}\left(B_{g}^{\dagger}\right)^{j}\left\{2 c(f, g)+4 N_{\bar{f} g}\right\}\left(B_{g}^{\dagger}\right)^{n-1-j} \Phi \\
& =2 c n(f, g)\left(B_{g}^{\dagger}\right)^{n-1} \Phi+4 \sum_{j=0}^{n-1}\left(B_{g}^{\dagger}\right)^{j} N_{\bar{f} g}\left(B_{g}^{\dagger}\right)^{n-1-j} \Phi
\end{aligned}
$$

By Lemma 1 we have

$$
N_{\bar{f} g}\left(B_{g}^{\dagger}\right)^{n-1-j} \Phi=2(n-1-j) B_{\bar{f} g^{2}}^{\dagger}\left(B_{g}^{\dagger}\right)^{n-2-j} \Phi
$$

Therefore,

$$
\begin{aligned}
\sum_{j=0}^{n-1}\left(B_{g}^{\dagger}\right)^{j} N_{\bar{f} g}\left(B_{g}^{\dagger}\right)^{n-1-j} \Phi & =\sum_{j=0}^{n-1}\left(B_{g}^{\dagger}\right)^{j} 2(n-1-j) B_{\bar{f} g^{2}}^{\dagger}\left(B_{g}^{\dagger}\right)^{n-2-j} \Phi \\
& =2 \sum_{j=0}^{n-1}(n-1-j) B_{\bar{f} g^{2}}^{\dagger}\left(B_{g}^{\dagger}\right)^{n-2} \Phi \\
& =n(n-1) B_{\bar{f} g^{2}}^{\dagger}\left(B_{g}^{\dagger}\right)^{n-2} \Phi
\end{aligned}
$$

We then use Equations (5), (6), and (7) to get

$$
\begin{aligned}
B_{f} \psi(g) & =\sum_{n=0}^{\infty} \frac{1}{n !} B_{f}\left(B_{g}^{\dagger}\right)^{n} \Phi \\
& =\sum_{n=1}^{\infty} \frac{1}{n !} 2 c n(f, g)\left(B_{g}^{\dagger}\right)^{n-1} \Phi+\sum_{n=2}^{\infty} \frac{1}{n !} 4 n(n-1) B_{\bar{f} g^{2}}^{\dagger}\left(B_{g}^{\dagger}\right)^{n-2} \Phi \\
& =2 c(f, g) \psi(g)+4 B_{\bar{f} g^{2}}^{\dagger} \psi(g) .
\end{aligned}
$$

This yields the lemma since $4 B_{\bar{f} g^{2}}^{\dagger} \psi(g)=2 N_{\bar{f} g} \psi(g)$ by Lemma 2 .

Lemma 4. The following equality holds:

$$
B_{f} B_{f} \psi(g)=\left\{4 c^{2}(f, g)^{2}+8 c\left(f^{2} \bar{g}, g\right)+8 c(f, g) N_{\bar{f} g}+8 N_{\bar{f}^{2} g^{2}}+4 N_{\bar{f} g}^{2}\right\} \psi(g)
$$

Proof. Apply Lemma 3 to obtain

$$
B_{f} B_{f} \psi(g)=\left\{4 c^{2}(f, g)^{2}+4 c(f, g) N_{\bar{f} g}+2 B_{f} N_{\bar{f} g}\right\} \psi(g) .
$$


By Equation (3) and Lemma 3 we have

$$
\begin{aligned}
B_{f} N_{\bar{f} g} \psi(g) & =\left\{\left[B_{f}, N_{\bar{f} g}\right]+N_{\bar{f} g} B_{f}\right\} \psi(g)=2 B_{f^{2} \bar{g}} \psi(g)+N_{\bar{f} g} B_{f} \psi(g) \\
& =\left\{4 c\left(f^{2} \bar{g}, g\right)+4 N_{\bar{f}^{2} g^{2}}+2 c(f, g) N_{\bar{f} g}+2 N_{\bar{f} g}^{2}\right\} \psi(g) .
\end{aligned}
$$

Upon putting Equation (9) into Equation (8) we get the equality in the lemma.

Lemma 5. $N_{f} N_{f} \psi(g)=\left\{4 B_{f^{2} g}^{\dagger}+4\left(B_{f g}^{\dagger}\right)^{2}\right\} \psi(g)$.

Proof. Use Lemma 2 and Equation (2) to get

$$
N_{f} N_{f} \psi(g)=2 N_{f} B_{f g}^{\dagger} \psi(g)=2\left\{\left[N_{f}, B_{f g}^{\dagger}\right]+B_{f g}^{\dagger} N_{f}\right\} \psi(g)=4 B_{f^{2} g}^{\dagger} \psi(g)+4\left(B_{f g}^{\dagger}\right)^{2} \psi(g)
$$

Now consider the operator valued measures on $\mathbf{R}$ defined by

$$
(s, t) \mapsto B_{\chi_{(s, t)}}, B_{\chi_{(s, t)}}^{\dagger}, N_{\chi_{(s, t)}}
$$

where

$$
\chi_{(s, t)}(r)=\left\{\begin{array}{lll}
1 & \text { if } & r \in(s, t) \\
0 & \text { if } & r \notin(s, t)
\end{array}\right.
$$

Notice that

$$
B_{\chi_{(s, t)}}=B_{\chi_{[s, t]}}=B_{\chi_{[s, t)}}=B_{\chi_{(s, t]}}
$$

and similar identities hold for the other operators. From now on all the test functions of exponential vectors will be supposed to be step functions of the form

$$
\sum_{j=1}^{\infty} g\left(t_{j}\right) \chi_{\left[t_{j}, t_{j+1}\right)}
$$

with $0<t_{1}<\ldots<t_{j}<\ldots$ and $g\left(t_{j}\right) \neq 0$ only in a finite number of points. Moreover we further restrict the test functions by the condition

$$
\|g\|_{\infty}=\sup _{t \in \mathbf{R}}|g(t)|<1 / 2
$$

It is known that the exponential vectors, corresponding to these test functions are total in the SWN space. We will use the notation

$$
d B_{t}=B_{\chi_{[t, t+d t)}} \quad ; \quad d B_{t}^{\dagger}=B_{\chi_{[t, t+d t)}^{\dagger}}^{\dagger} \quad ; \quad d N_{t}=N_{\chi_{[t, t+d t)}}
$$

where $t, d t>0$ and in all expressions of the form

$$
\left\langle\psi(f), d H_{t} d K_{t} \psi(g)\right\rangle
$$


$\left(H, K=B, B^{+}, N\right)$ the number $d t$ is supposed to be such that both functions $f, g$ are constant in the interval $[t, t+d t]$ for all $t \in \mathbf{R}$ with the exception of at most a finite number of points. With this convenction we have that

$$
\left\langle\chi_{[t, t+d t]}, g\right\rangle=g(t) d t
$$

for almost all $t \in \mathbf{R}$ with respect to the Lebesgue measure.

Now, we introduce a notation. Let $F(x, y)=\sum_{m, n \geq 0} a_{m, n} x^{m} y^{n}$ be an analytic function. Define $F\left(\partial_{t}^{*}, \partial_{t}\right)$ by

$$
\left\langle\psi(f), M F\left(\partial_{t}^{*}, \partial_{t}\right) \psi(g)=\left\langle\psi(f), F\left(\partial_{t}^{*}, \partial_{t}\right) M \psi(g)\right\rangle=\sum_{m, n \geq 0} a_{m, n} \bar{f}(t)^{m} g(t)^{n}\langle\psi(f), M \psi(g)\rangle,\right.
$$

where $M$ is any operator in the algebra generated by $B_{f}, B_{f}^{\dagger}, N_{f}$. We remark that, in the case of 1-st order white noise, $\partial_{t}$ and $\partial_{t}^{*}$ are the Hida derivative (or the white noise differential operator) and its adjoint (see the books [6] [7].)

Equation (10) defines the symbols $F\left(\partial_{t}^{*}, \partial_{t}\right) M, M F\left(\partial_{t}^{*}, \partial_{t}\right)$ as sesquilinear forms on the vector space algebraically spanned by the SWN exponential vectors. By construction $M F\left(\partial_{t}^{*}, \partial_{t}\right)=F\left(\partial_{t}^{*}, \partial_{t}\right) M$ in the sense of quadratic forms for all $M$ in the algebra generated by $B_{f}, B_{f}^{\dagger}, N_{f}$. In particular $\partial_{t} M=M \partial_{t}$ and $\partial_{t}^{*} M=M \partial^{*}$ in the sense of sesquilinear forms. Moreover, we have the following lemma.

Lemma 6. The equality $\partial_{t}^{*} d B_{t}=\partial_{t} d B_{t}^{\dagger}$ holds in the sense of sesquilinear forms on the algebraic span of the exponential vectors.

Proof. From Lemma 3 we get $d B_{t} \psi(g)=\left(2 c g(t) d t+2 g(t) d N_{t}\right) \psi(g)$. Hence

$$
\begin{aligned}
\left\langle\psi(f), \partial_{t}^{*} d B_{t} \psi(g)\right\rangle & =\bar{f}(t)\left\langle\psi(f), d B_{t} \psi(g)\right\rangle=\bar{f}(t)\left\langle\psi(f),\left(2 c g(t) d t+2 g(t) d N_{t}\right) \psi(g)\right\rangle \\
& =\bar{f}(t) g(t)\left\langle\psi(f),\left(2 c d t+2 d N_{t}\right) \psi(g)\right\rangle
\end{aligned}
$$

On the other hand, we have

$$
\begin{aligned}
\left\langle\psi(f), \partial_{t} d B_{t}^{\dagger} \psi(g)\right\rangle & =g(t)\left\langle\psi(f), d B_{t}^{\dagger} \psi(g)\right\rangle=g(t)\left\langle d B_{t} \psi(f), \psi(g)\right\rangle \\
& =g(t)\left\langle\left(2 c f(t) d t+2 f(t) d N_{t}\right) \psi(f), \psi(g)\right\rangle \\
& =\bar{f}(t) g(t)\left\langle\left(2 c d t+2 d N_{t}\right) \psi(f), \psi(g)\right\rangle
\end{aligned}
$$

Since $c$ is a real number, the lemma follows from Equations (11) and (12).

Now, apply Lemma 4 to the function $f=\chi_{[t, t+d t)}=d \chi_{t}$ to get

$$
B_{d \chi_{t}} B_{d \chi_{t}} \psi(g)=\left\{8 c g(t)^{2} d t+8 g(t)^{2} N_{d \chi_{t}}+4 g(t)^{2} N_{d \chi_{t}}^{2}\right\} \psi(g),
$$

which can be written in another form:

$$
d B_{t} d B_{t} \psi(g)=\left\{8 c g(t)^{2} d t+8 g(t)^{2} d N_{t}+4 g(t)^{2}\left(d N_{t}\right)^{2}\right\} \psi(g)
$$


By using the notation in Equation (10) we can rewrite Equation (13) as follows:

$$
d B_{t} d B_{t}=8 c \partial_{t}^{2} d t+8 \partial_{t}^{2} d N_{t}+4 \partial_{t}^{2}\left(d N_{t}\right)^{2}
$$

This equation is understood to hold weakly on exponential vectors $\psi(g)$.

Apply Lemma 5 to the function $f=\chi_{[t, t+d t)}=d \chi_{t}$ to get

$$
d N_{t} d N_{t} \psi(g)=\left\{4 g(t) d B_{t}^{\dagger}+4 g(t)^{2}\left(d B_{t}^{\dagger}\right)^{2}\right\} \psi(g)
$$

Therefore, with the notation in Equation (10), the following equality holds weakly on exponential vectors

$$
d N_{t} d N_{t}=4 \partial_{t} d B_{t}^{\dagger}+4 \partial_{t}^{2}\left(d B_{t}^{\dagger}\right)^{2}
$$

Next, consider $d B_{t}^{\dagger} d B_{t}^{\dagger}$. We can use Equation (13) to derive

$$
\begin{aligned}
\left\langle\psi(f), d B_{t}^{\dagger} d B_{t}^{\dagger} \psi(g)\right\rangle & =\left\langle d B_{t} d B_{t} \psi(f), \psi(g)\right\rangle \\
& =\left\langle\left\{8 c f(t)^{2} d t+8 f(t)^{2} d N_{t}+4 f(t)^{2}\left(d N_{t}\right)^{2}\right\} \psi(f), \psi(g)\right\rangle \\
& =\bar{f}(t)^{2}\left\langle\left\{8 c d t+8 d N_{t}+4\left(d N_{t}\right)^{2}\right\} \psi(f), \psi(g)\right\rangle \\
& =\bar{f}(t)^{2}\left\langle\psi(f),\left\{8 c d t+8 d N_{t}+4\left(d N_{t}\right)^{2}\right\} \psi(g)\right\rangle \\
& =\left\langle\psi(f),\left(\partial_{t}^{*}\right)^{2}\left\{8 c d t+8 d N_{t}+4\left(d N_{t}\right)^{2}\right\} \psi(g)\right\rangle .
\end{aligned}
$$

Therefore, we get the following equality

$$
d B_{t}^{\dagger} d B_{t}^{\dagger}=8 c\left(\partial_{t}^{*}\right)^{2} d t+8\left(\partial_{t}^{*}\right)^{2} d N_{t}+4\left(\partial_{t}^{*}\right)^{2}\left(d N_{t}\right)^{2}
$$

Equations (14), (15), (16) are linear in the quadratic differentials. By solving them we easily derive

$$
\begin{aligned}
& d N_{t} d N_{t}=\frac{32 c\left(\partial_{t}^{*}\right)^{2} \partial_{t}^{2}}{1-16\left(\partial_{t}^{*}\right)^{2} \partial_{t}^{2}} d t+\frac{4 \partial_{t}}{1-16\left(\partial_{t}^{*}\right)^{2} \partial_{t}^{2}} d B_{t}^{\dagger}+\frac{32\left(\partial_{t}^{*}\right)^{2} \partial_{t}^{2}}{1-16\left(\partial_{t}^{*}\right)^{2} \partial_{t}^{2}} d N_{t} . \\
& d B_{t} d B_{t}=\frac{8 c \partial_{t}^{2}}{1-16\left(\partial_{t}^{*}\right)^{2} \partial_{t}^{2}} d t+\frac{16 \partial_{t}^{3}}{1-16\left(\partial_{t}^{*}\right)^{2} \partial_{t}^{2}} d B_{t}^{\dagger}+\frac{8 \partial_{t}^{2}}{1-16\left(\partial_{t}^{*}\right)^{2} \partial_{t}^{2}} d N_{t}, \\
& d B_{t}^{\dagger} d B_{t}^{\dagger}=\frac{8 c\left(\partial_{t}^{*}\right)^{2}}{1-16\left(\partial_{t}^{*}\right)^{2} \partial_{t}^{2}} d t+\frac{16\left(\partial_{t}^{*}\right)^{2} \partial_{t}}{1-16\left(\partial_{t}^{*}\right)^{2} \partial_{t}^{2}} d B_{t}^{\dagger}+\frac{8\left(\partial_{t}^{*}\right)^{2}}{1-16\left(\partial_{t}^{*}\right)^{2} \partial_{t}^{2}} d N_{t},
\end{aligned}
$$

and, using Lemma 6 we find

$$
d B_{t}^{\dagger} d B_{t}^{\dagger}=\frac{8 c\left(\partial_{t}^{*}\right)^{2}}{1-16\left(\partial_{t}^{*}\right)^{2} \partial_{t}^{2}} d t+\frac{16\left(\partial_{t}^{*}\right)^{3}}{1-16\left(\partial_{t}^{*}\right)^{2} \partial_{t}^{2}} d B_{t}+\frac{8\left(\partial_{t}^{*}\right)^{2}}{1-16\left(\partial_{t}^{*}\right)^{2} \partial_{t}^{2}} d N_{t}
$$

which is obtained by taking the formal adjoint of both sides of (18) and conversely the adjoint of the right hand side of Equation (19) is the right hand side of Equation (18). 
Notice that, because of Lemma 6, the right hand side of (19) is formally self-adjoint, as it should be. Next, by Lemma 3 we have

$$
N_{f} B_{f} \psi(g)=\left(2 c(f, g) N_{f}+2 N_{f} N_{\bar{f} g}\right) \psi(g) .
$$

Then take $f=\chi_{[t, t+d t)}=: d \chi_{t}$ and use the fact $d t d N_{t}=0$ in the sense that, for any uniformly bounded function $F(t)$ and for any pair of exponential vectors $\psi(f), \psi(g)$ one has:

$$
\lim _{\max \left(t_{j+1}-t_{j}\right) \rightarrow 0} \sum_{j=1}^{N}\left\langle\psi(f), F\left(t_{j}\right)\left(t_{i+1}-t_{j}\right) N_{\chi_{\left(t_{j}, t_{j+1}\right)}} \psi(g)\right\rangle=0
$$

to get

$$
N_{d \chi_{t}} B_{d \chi_{t}} \psi(g)=2 g(t)\left(N_{d \chi_{t}}\right)^{2} \psi(g),
$$

which, using the notation (10), can be expressed as

$$
d N_{t} d B_{t}=2 \partial_{t}\left(d N_{t}\right)^{2}
$$

Therefore, by Equation (17) we obtain the following equality,

$$
d N_{t} d B_{t}=\frac{64 c\left(\partial_{t}^{*}\right)^{2} \partial_{t}^{3}}{1-16\left(\partial_{t}^{*}\right)^{2} \partial_{t}^{2}} d t+\frac{8 \partial_{t}^{2}}{1-16\left(\partial_{t}^{*}\right)^{2} \partial_{t}^{2}} d B_{t}^{\dagger}+\frac{64\left(\partial_{t}^{*}\right)^{2} \partial_{t}^{3}}{1-16\left(\partial_{t}^{*}\right)^{2} \partial_{t}^{2}} d N_{t}
$$

Next, use Lemma 3 to get

$$
B_{f}^{\dagger} B_{f} \psi(g)=2 c(f, g) B_{f}^{\dagger} \psi(g)+2 B_{f}^{\dagger} N_{\bar{f} g} \psi(g)
$$

Take $f=\chi_{[t, t+d t)}=d \chi_{t}$ and note that, in the sense explained above, $d t d B_{t}^{\dagger}=0, d B_{t}^{\dagger} d N_{t}=$ $2 \partial_{t}^{*}\left(d N_{t}\right)^{2}$. Hence

$$
d B_{t}^{\dagger} d B_{t}=2 \partial_{t} d B_{t}^{\dagger} d N_{t}=4 \partial_{t}^{*} \partial_{t}\left(d N_{t}\right)^{2}
$$

Therefore, by Equation (17),

$$
d B_{t}^{\dagger} d B_{t}=\frac{128 c\left(\partial_{t}^{*}\right)^{3} \partial_{t}^{3}}{1-16\left(\partial_{t}^{*}\right)^{2} \partial_{t}^{2}} d t+\frac{16 \partial_{t}^{*} \partial_{t}^{2}}{1-16\left(\partial_{t}^{*}\right)^{2} \partial_{t}^{2}} d B_{t}^{\dagger}+\frac{128\left(\partial_{t}^{*}\right)^{3} \partial_{t}^{3}}{1-16\left(\partial_{t}^{*}\right)^{2} \partial_{t}^{2}} d N_{t}
$$

To find $d B_{t} d B_{t}^{\dagger}$, note that by Equation (1) we have

$$
B_{f} B_{f}^{\dagger}=\left[B_{f}, B_{f}^{\dagger}\right]+B_{f}^{\dagger} B_{f}=2 c(f, f)+4 N_{\bar{f} f}+B_{f}^{\dagger} B_{f} .
$$

Take $f=\chi_{[t, t+d t)}=d \chi_{t}$ and then apply Equation (21) to get

$$
\begin{aligned}
d B_{t} d B_{t}^{\dagger}= & \frac{2 c-32 c\left(\partial_{t}^{*}\right)^{2} \partial_{t}^{2}+128 c\left(\partial_{t}^{*}\right)^{3} \partial_{t}^{3}}{1-16\left(\partial_{t}^{*}\right)^{2} \partial_{t}^{2}} d t+\frac{16 \partial_{t}^{*} \partial_{t}^{2}}{1-16\left(\partial_{t}^{*}\right)^{2} \partial_{t}^{2}} d B_{t}^{\dagger} \\
& +\frac{4-64\left(\partial_{t}^{*}\right)^{2} \partial_{t}^{2}+128\left(\partial_{t}^{*}\right)^{3} \partial_{t}^{3}}{1-16\left(\partial_{t}^{*}\right)^{2} \partial_{t}^{2}} d N_{t}
\end{aligned}
$$


Now, note that $B_{f} N_{f}=-\left[N_{f}, B_{f}\right]+N_{f} B_{f}=2 B_{\bar{f} f}+N_{f} B_{f}$ by Equation (3). Take $f=\chi_{[t, t+d t)}=d \chi_{t}$ to get

$$
d B_{t} d N_{t}=2 d B_{t}+d N_{t} d B_{t}
$$

Hence by Equation (20) we obtain the equality

$$
d B_{t} d N_{t}=\frac{64 c\left(\partial_{t}^{*}\right)^{2} \partial_{t}^{3}}{1-16\left(\partial_{t}^{*}\right)^{2} \partial_{t}^{2}} d t+2 d B_{t}+\frac{8 \partial_{t}^{2}}{1-16\left(\partial_{t}^{*}\right)^{2} \partial_{t}^{2}} d B_{t}^{\dagger}+\frac{64\left(\partial_{t}^{*}\right)^{2} \partial_{t}^{3}}{1-16\left(\partial_{t}^{*}\right)^{2} \partial_{t}^{2}} d N_{t}
$$

which, by Lemma 6 , can be rewritten as

$$
d B_{t} d N_{t}=\frac{64 c\left(\partial_{t}^{*}\right)^{2} \partial_{t}^{3}}{1-16\left(\partial_{t}^{*}\right)^{2} \partial_{t}^{2}} d t+\left\{\frac{2 \partial_{t}}{\partial_{t}^{*}}+\frac{8 \partial_{t}^{2}}{1-16\left(\partial_{t}^{*}\right)^{2} \partial_{t}^{2}}\right\} d B_{t}^{\dagger}+\frac{64\left(\partial_{t}^{*}\right)^{2} \partial_{t}^{3}}{1-16\left(\partial_{t}^{*}\right)^{2} \partial_{t}^{2}} d N_{t}
$$

From Equation (23), using the identity

$$
\left\langle\psi(f), d N_{t} d B_{t}^{\dagger} \psi(g)\right\rangle=\overline{\left\langle\psi(g), d B_{t} d N_{t} \psi(f)\right\rangle}
$$

we can derive the equality:

$$
d N_{t} d B_{t}^{\dagger}=\frac{64 c\left(\partial_{t}^{*}\right)^{3} \partial_{t}^{2}}{1-16\left(\partial_{t}^{*}\right)^{2} \partial_{t}^{2}} d t+\frac{8\left(\partial_{t}^{*}\right)^{2}}{1-16\left(\partial_{t}^{*}\right)^{2} \partial_{t}^{2}} d B_{t}+2 d B_{t}^{\dagger}+\frac{64\left(\partial_{t}^{*}\right)^{3} \partial_{t}^{2}}{1-16\left(\partial_{t}^{*}\right)^{2} \partial_{t}^{2}} d N_{t}
$$

which, by Lemma 6, can be rewritten as

$$
d N_{t} d B_{t}^{\dagger}=\frac{64 c\left(\partial_{t}^{*}\right)^{3} \partial_{t}^{2}}{1-16\left(\partial_{t}^{*}\right)^{2} \partial_{t}^{2}} d t+\left\{2+\frac{8 \partial_{t}^{*} \partial_{t}}{1-16\left(\partial_{t}^{*}\right) \partial_{t}^{2}}\right\} d B_{t}^{\dagger}+\frac{64\left(\partial_{t}^{*}\right)^{3} \partial_{t}^{2}}{1-16\left(\partial_{t}^{*}\right)^{2} \partial_{t}^{2}} d N_{t}
$$

Notice that (25) is obtained from (23) by taking the formal adjoint and conversely (23) is obtained from (25) in the same way. Finally, use Equation (2) to get

$$
B_{f}^{\dagger} N_{f}=-\left[N_{f}, B_{f}^{\dagger}\right]+N_{f} B_{f}^{\dagger}=-2 B_{f^{2}}^{\dagger}+N_{f} B_{f}^{\dagger}
$$

Then take $f=\chi_{[t, t+d t]}=d \chi_{t}$ to show that

$$
d B_{t}^{\dagger} d N_{t}=-2 d B_{t}^{\dagger}+d N_{t} d B_{t}^{\dagger}
$$

Hence by Equation (25) we have

$$
d B_{t}^{\dagger} d N_{t}=\frac{64 c\left(\partial_{t}^{*}\right)^{3} \partial_{t}^{2}}{1-16\left(\partial_{t}^{*}\right)^{2} \partial_{t}^{2}} d t+\frac{8\left(\partial_{t}^{*}\right)^{2}}{1-16\left(\partial_{t}^{*}\right)^{2} \partial_{t}^{2}} d B_{t}+\frac{64\left(\partial_{t}^{*}\right)^{3} \partial_{t}^{2}}{1-16\left(\partial_{t}^{*}\right)^{2} \partial_{t}^{2}} d N_{t}
$$

which, by Lemma 6 , can be rewritten as

$$
d B_{t}^{\dagger} d N_{t}=\frac{64 c\left(\partial_{t}^{*}\right)^{3} \partial_{t}^{2}}{1-16\left(\partial_{t}^{*}\right)^{2} \partial_{t}^{2}} d t+\frac{8 \partial_{t}^{*} \partial_{t}}{1-16\left(\partial_{t}^{*}\right)^{2} \partial_{t}^{2}} d B_{t}^{\dagger}+\frac{64\left(\partial_{t}^{*}\right)^{3} \partial_{t}^{2}}{1-16\left(\partial_{t}^{*}\right)^{2} \partial_{t}^{2}} d N_{t}
$$

Summing up, we have the following: 
Theorem (2). The Itô table for the renormalized square of white noise is given by

\begin{tabular}{|l|l|l|l|}
\hline & $d B_{t}^{\dagger}$ & $d N_{t}$ & $d B_{t}$ \\
\hline$d B_{t}$ & $\alpha_{-}^{+} d t+\beta_{-}^{+} d B_{t}^{\dagger}+\gamma_{-}^{+} d N_{t}$ & $\alpha_{-}^{0} d t+\beta_{-}^{0} d B_{t}^{\dagger}+\gamma_{-}^{0} d N_{t}$ & $\alpha_{-}^{-} d t+\beta_{-}^{-} d B_{t}^{\dagger}+\gamma_{-}^{-} d N_{t}$ \\
\hline$d N_{t}$ & $\alpha_{0}^{+} d t+\beta_{0}^{+} d B_{t}^{\dagger}+\gamma_{0}^{+} d N_{t}$ & $\alpha_{0}^{0} d t+\beta_{0}^{0} d B_{t}^{\dagger}+\gamma_{0}^{0} d N_{t}$ & $\alpha_{0}^{-} d t+\beta_{0}^{-} d B_{t}^{\dagger}+\gamma_{0}^{-} d N_{t}$ \\
\hline$d B_{t}^{\dagger}$ & $\alpha_{+}^{+} d t+\beta_{+}^{+} d B_{t}^{\dagger}+\gamma_{+}^{+} d N_{t}$ & $\alpha_{+}^{0} d t+\beta_{+}^{0} d B_{t}^{\dagger}+\gamma_{+}^{0} d N_{t}$ & $\alpha_{+}^{-} d t+\beta_{+}^{-} d B_{t}^{\dagger}+\gamma_{+}^{-} d N_{t}$ \\
\hline
\end{tabular}

where the coefficients $\alpha_{\varepsilon}^{\varepsilon^{\prime}}, \beta_{\varepsilon}^{\varepsilon^{\prime}}, \gamma_{\varepsilon}^{\varepsilon^{\prime}},\left(\varepsilon, \varepsilon^{\prime} \in\{+,-, 0\}\right)$ are given by equations (17) (18) (19) (20) (21) (22) (24) (26) (28).

Acknowledgements. The results in this paper were obtained during the visits of T. Hida and H.-H. Kuo to the Centro Vito Volterra (CVV), Università degli Studi di Roma "Tor Vergata" (June20-July 20, 2000 for TH and May 24-July 31, 2000 for HHK.) They want to thank CVV for the financial support and to give their deepest appreciation to Professors L. Accardi and R. Monte, for the warm hospitality during their visits.

\section{REFERENCES}

[1] Accardi, L., Franz, U., and Skeide, M.: Renormalized squares of white noise and other non-Gaussian noises as Lévy processes on real Lie algebras; Centro Vito Volterra, Università di Roma "Tor Vergata" Preprint \#423 (2000)

[2] Accardi, L., Lu, Y. G., and Volovich, I. V.: Non-linear extensions of classical and quantum stochastic calculus and essentially infinite dimensional analysis; Lecture Notes in Statistics, Springer-Verlag 128 (1998) 1-33

[3] Accardi, L., Lu, Y. G., and Volovich, I. V.: White noise approach to classical and quantum stochastic calculi; Centro Vito Volterra, Università di Roma "Tor Vergata" Preprint \#375 (1999)

[4] Accardi, L., Lu, Y. G., and Volovich, I. V.: A white noise approach to stochastic calculus; Centro Vito Volterra, Università di Roma "Tor Vergata" Preprint \#417 (2000)

[5] Accardi, L. and Skeide, M.: On the relation of the square of white noise and the finite difference algebra; Infinite Dimensional Analysis, Quantum Probability and Related Topics 3 (2000) 185-189

[6] Hida, T., Kuo, H.-H., Potthoff, J., and Streit, L.: White Noise: An Infinite Dimensional Calculus. Kluwer Academic Publishers, 1993

[7] Kuo, H.-H.: White Noise Distribution Theory. CRC Press, Boca Raton, 1996

[8] Śniady P. Quadratic bosonic and free white noises. Commun. Math. Phys., 211 (3) (2000) 615-628

[9] L. Accardi, A. Boukas: The semimartigale property of the square of white noise integrators; Centro Vito Volterra, Università di Roma "Tor Vergata" Preprint \#429 (2000). 